Thorax (1963), 18, 291

\title{
Neuropathological findings in patients dying after open-heart surgery
}

\author{
J . B . B R I E R L E Y \\ From the Department of Neuropathology, Institute of Psychiatry, London
}

Although cerebral damage has been regarded as the cause of death in some patients dying after open operations on the heart, this conclusion rests almost entirely on clinical evidence such as an impaired level of consciousness after the operation, abnormalities of muscle tone and reflexes, visual and extrapyramidal disorders or epilepsy.

Accounts of cerebral pathology in fatal cases are limited to that of Björk and Hultquist (1960) and a group of papers describing the cerebral emboli due to the anti-foam agent employed in the oxygenator (Yates, Cassie, Dark, Jack, and Riddell, 1959 ; Smith, 1960 ; Lindberg, Lucas, Sheagren, and Malm, 1961 ; Thomassen, Howbert, Winn, and Thompson, 1961).

In the 11 patients reported by Björk and Hultquist, death was attributed to cerebral damage in eight, all children. As yet there appears to have been no detailed pathological study of the brains of adults dying from presumed cerebral damage.

Here we present the neuropathological findings in the first 11 of a series of patients who died from six and a half hours to 11 days after open cardiac surgery. The brains, received from six cardiac surgery units, were from patients 10 to 51 years of age, and all but one were subjected to some degree of hypothermia.

The cases are divided into two groups: Group I, two cases in which cardio-pulmonary bypass was not employed and in which there was deliberate circulatory arrest under mild hypothermia ; and Group II, nine cases in which cardio-pulmonary bypass was effected by a pump oxygenator. Mild to moderate hypothermia was employed in eight, being produced by a heat exchanger in seven and by surface cooling in one. There was no deliberate arrest of the circulation by interruption of the perfusion.

\section{MATERIAL AND METHODS}

Unless previously dissected, the brains were cut into coronal slices after removal of the brain stem and cerebellum. Blocks were taken from the temporal lobes (to include basal ganglia), frontal and occipital lobes, midbrain, pons, medulla, and cerebellum and embedded in nitrocellulose. Sections were stained with haematoxylin and eosin, haematoxylin and Van Gieson's counterstain, the method of Nissl (using cresyl violet), with Mallory's phosphotungstic acid haematoxylin, with Heidenhain's haematoxylin, and by the periodic acid Schiff technique.

Frozen sections were stained with Oil Red O.

\section{SUMMARIES OF CASES IN GROUP I}

THE principal cerebral structures referred to in the text are indicated in Diagrams 1 and 2.

CASE 1 (Maudsley No. 1339) A boy aged 16 years had congenital pulmonary stenosis without septal defect.

Operation Cooling was obtained by the venovenous technique of Ross (1954) from $36^{\circ}$ to $31^{\circ} \mathrm{C}$. in 27 minutes. The heart was arrested for $4 \mathrm{~min} .20 \mathrm{sec}$. and the valve was incised. Rewarming to $33.2^{\circ} \mathrm{C}$. took 12 minutes. The heart re-started satisfactorily and the blood pressure was normal.

Post-operative course There were signs of extreme cerebral irritation on leaving the theatre, and so intravenous urea and sucrose were given, but without effect. Athetoid movements were noted and five leftsided fits occurred within an hour. Tracheostomy and assisted respiration became necessary and surface cooling to $32^{\circ} \mathrm{C}$. was carried out. On the first postoperative day the patient was unconscious. A right frontal burr hole excluded a subdural haematoma. On the third post-operative day an anuric regime was begun and was successful. On the seventh postoperative day athetoid movements of the right arm and leg were noted and there was gradual deterioration to death on the tenth post-operative day.

Necropsy Some antemortem thrombus was seen in the inferior vena cava and portal vein; there was also altered blood in the small and large intestines.

Macroscopic examination of the brain showed slight convolutional flattening and considerable congestion both most marked over the occipital lobes. A thin film of subarachnoid blood covered the right occipital lobe. There were no internal herniae and the basal vessels were normal.

The right hemisphere was slightly expanded and its ventricles small. A recent haemorrhage $(1.0 \mathrm{~cm}$. diameter) was noted in the white matter of the left 


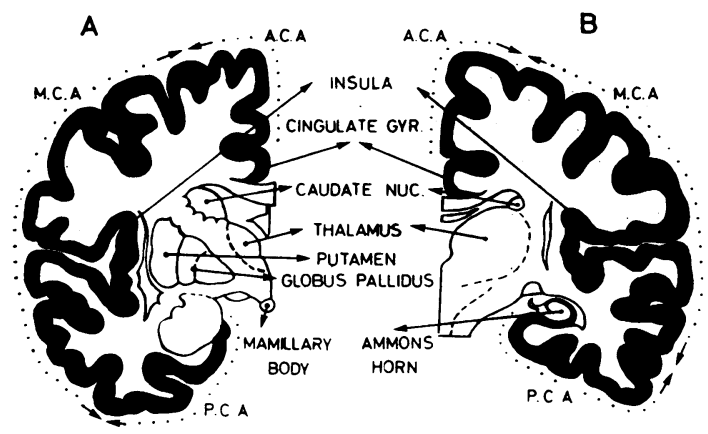

DIAGRAM 1. Cerebral hemispheres: two coronal levels indicating structures referred to in text. A. Through basal ganglia and hypothalamus. $B$. Through thalamus and Ammon's horn. A.C.A., M.C.A., and P.C.A.=cortical areas supplied by anterior, middle, and posterior cerebral arteries. Note. Diencephalon=structures around the third ventricle, i.e., thalamus, subthalamic region, and hypothalamus.

parietal lobe. The cortical ribbon on the right showed well-defined quadrilateral or irregular patches of discolouration, which were soft to the touch (Fig. 1), and the right caudate nucleus and putamen showed similar areas.

Microscopic examination of the brain showed changes which were both diffuse and localized. The latter were the more conspicuous and were present throughout the right hemisphere but maximal in the occipital lobe. On the left, they were confined to the occipital lobe.

The localized changes appeared as areas of variable neuronal loss which were perivascular (focal) and also large, rectangular or irregular ('geographical') (Fig. 2). Many of the latter were surrounded by a narrow band of spongy tissue and corresponded to the discoloured areas seen on naked-eye examination.

The calcarine cortex was involved on both sides, and in the right occipital lobe the damage, which was largely on the lateral aspect, decreased anteriorly.

The hippocampus (Ammon's horn) was normal on the left but on the right showed a strip of neuronal necrosis in the deeper part of the Sommer sector (H. 1.). Geographical lesions in the caudate nucleus and putamen were the only other abnormalities in the grey matter. White matter damage consisted of a narrow band of partial demyelination deep to the damaged calcarine cortex.

Within the lesions there was ischaemic cell change. moderate glial proliferation, and occasional lipid phagocytes. There was no evidence of thrombosis or emboli (including fat) in the blood vessels.

Diffuse changes occurred outside the localized cortical lesions and there was much gliosis in the outer layers. There was a moderate reduction in cerebellar Purkinje cells and in the neurones of the inferior colliculi and mamillary bodies.
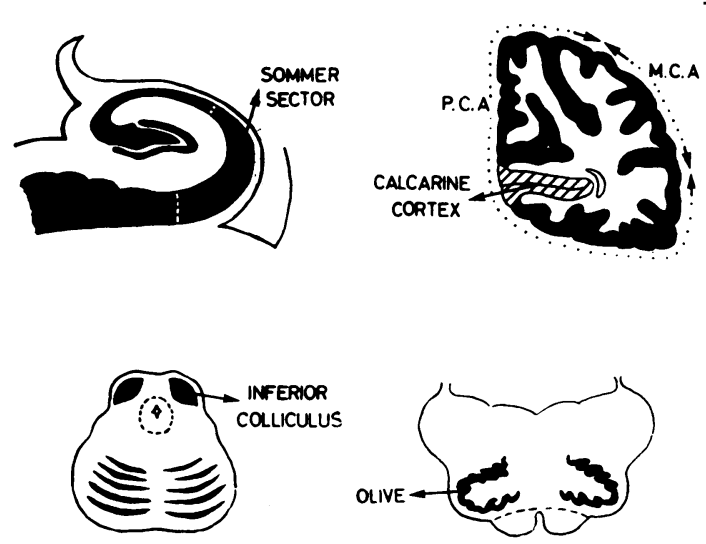

DIAGRAM 2. Coronal planes through Ammon's horn and occipital lobe, and transverse planes through midbrain and medulla (not to scale).

CASE 2 (Maudsley No. 1800) A woman aged 45 had a congenital atrial septal defect.

Operation After surface cooling to $28 \cdot 8^{\circ} \mathrm{C}$. thes heart was arrested for $5 \mathrm{~min} .37 \mathrm{sec}$. and the repair was effected. Ventricular fibrillation began on re warming, and cardiac massage was required for $60 \mathrm{D}$ min. before normal beating was resumed at temperature of $31.5^{\circ} \mathrm{C}$.

Post-operative course There was no recovery of consciousness. The blood pressure was $75 / 50 \mathrm{~mm}$. $\mathrm{Hg}$. Tracheostomy and assisted respiration became necessary and extensor plantar responses were noted. There was little change until death two days after theo operation.

LEGENDS TO PLATE OPPOSITE

FIG. 1. Case 1. Brain slice; frontal lobe, showing cleari. outlined patches of cortical discolouration. $(\times 3)$

FIG. 2. Case 1. Occipital lobe showing focal (perivascular $\mathrm{g}$ and irregular (geographical) areas of cell loss and pallor o $\overrightarrow{\beta_{7}}$ staining. (Cresyl violet, $\times 5$ )

FIG. 3. Cast 3. (Survival $6 \frac{1}{2}$ hours.) Occipital cortexin showing a pale staining band running across more darkl stained normal cortex. (P.A.S. and haematoxylin, $\times 28) \mathrm{N}$ FIG. 4. Case 3. Occipital cortex showing typical ischaemi£ change in a pyramidal neurone. The cell body is dark and shrunken and covered with fine particulate encrustations. (Cresyl violet, $\times 375$ )

FIG. 5. Case 4. Occipital lobe showing jurction betweer normal cortex (above) and a geographical lesion. In the latter, there is cell loss and shrinkage but normal glia (Cresyl violet,$\times 200)$

FIG. 6. Case 4. Teflon embolus in cortical vessel: (a) Haematoxylin and van Gieson $(\times 190) ;(b)$ polarized light $(\times 190)$. 

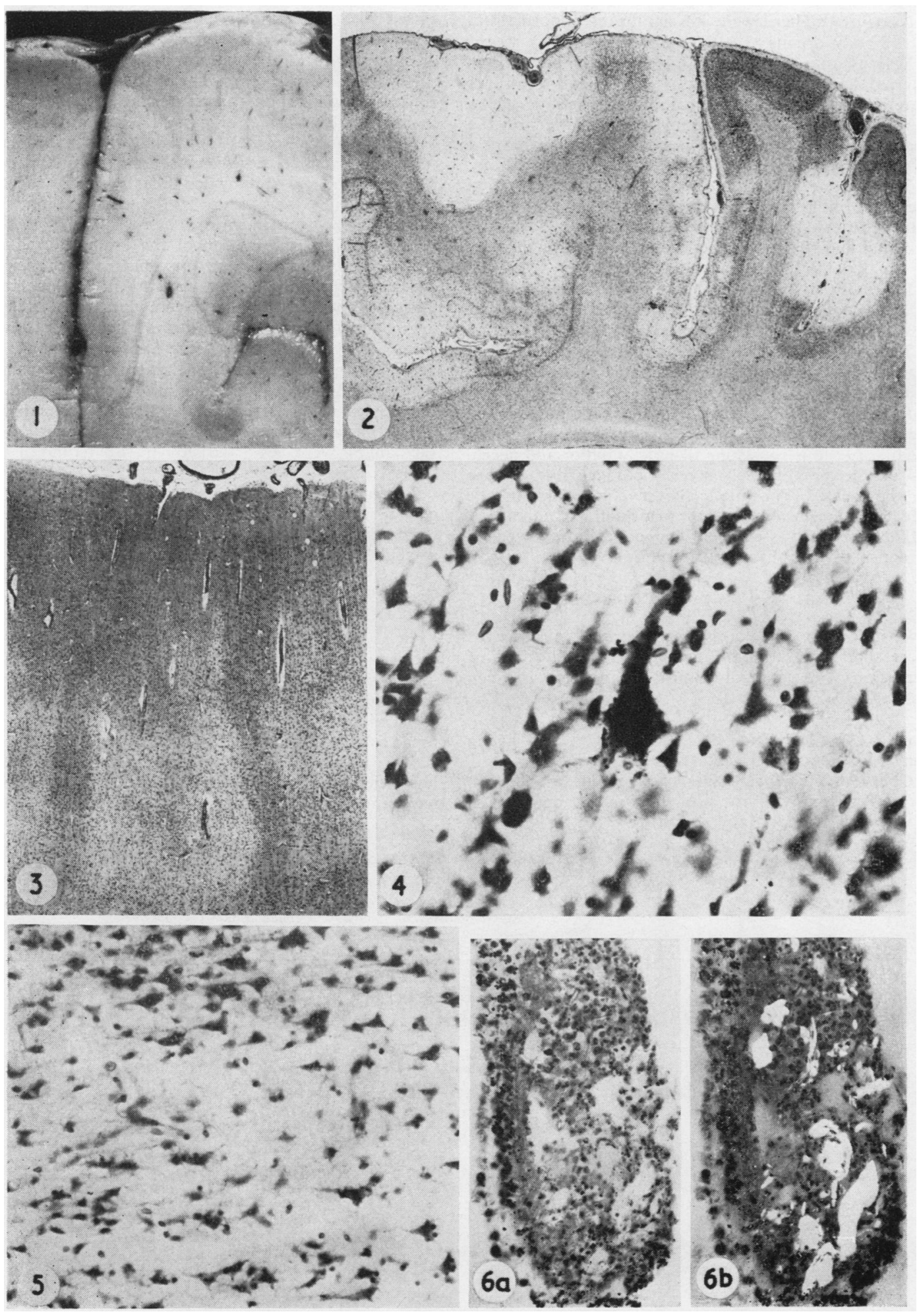
Necropsy There were no significant abnormalities in the body organs.

Macroscopic examination of the brain showed no internal herniae and the basal vessels and ventricles were normal. Areas of discolouration and petechiae were seen in the cortex and white matter of the occipital lobe, insula, and parietal and outer aspect of the temporal lobe, more on the left side throughout.

Microscopic examination of the brain showed in the cerebral hemispheres geographical areas of subtotal cortical necrosis in the outer aspect of the occipital and temporal lobes and lesser patchy damage in the frontal lobes ventrolaterally.

In the affected areas there was ischaemic cell change, moderate glio-mesodermal proliferation, and also islands of more or less intact cortex.

The Ammon's horns were normal and in the basal ganglia there was only patchy neuronal loss in the caudate nuclei.

In the cerebellum there was widespread homogenizing change in the Purkinje cells but there were also localized lesions consisting of patchy pallor and necrosis of the granule cell layer. homogenizing change in the related Purkinje cells, myelin pallor in the affected folia, and scattered petechial haemorrhages.

The cerebral vessels were congested but showed no evidence of embolism or thrombosis.

\section{SUMMARIFS OF CASES IN GROUP II}

The following nine cases are arranged in order of increasing survival time calculated from the end of perfusion. The essential clinical information is given in the Table.

CASE 3 (Maudsley No. 1892) A boy aged 10 had a congenital ventricular septal defect.

Operation Perfusion was carried out for $74 \mathrm{~min}$. at normal temperature and the septal defect was closed without a patch. Heavy haemorrhage occurreף when the aorta was unclamped and again of de-cannulation of the femoral artery.

Post-operative course The patient remained ur $\mathbb{B}$ conscious with spontaneous respiration until deatio from cardiac failure after six and a half hours.

Necropsy Only the lower halves of the hemp spheres were received without brain stem and cerebellum. Oval and rectangular areas of pallor togetheys with petechiae were seen in the occipital and less clearly in the frontal cortex.

Microscopic examination of the brain showed that the cortical changes were maximal in the occipita lobes, affecting the whole cortical ribbon includinig the calcarine region. There were numerous irregulaf or rectangular areas of pallor of neuronal staining (Fig. 3) within which the tissue appeared spongy. and neurones were shrunken and hyperchromatic as we $\vec{H}$ as exhibiting typical ischaemic cell change (Fig. 4D There was no glial reaction and there was capillar@ congestion and numerous ring haemorrhages.

Changes were less marked in the temporal and frontal lobes.

A narrow wedge of ischaemic cells was the onls change in the Ammon's horns. There were scattere $\$$ ischaemic neurones in the major thalamic nuclei anめ a slight diffuse pallor of myelin staining in the occipital lobe. No emboli were seen in the cerebra vessels.

CASE 4 (Maudsley No. 1689) A woman aged 29 haæ a congenital atrial septal defect and cleft mitral valv $\overrightarrow{5}$

Operation Perfusion was carried out for $107 \mathrm{mim}$ at a minimum temperature of $30 \cdot 4^{\circ} \mathrm{C}$. The defect was repaired with a Teflon patch and the mitral valve was repaired. The electronic pacemaker was applied (rate $72 / \mathrm{min}$.) and the systolic blood pressure was 60 कृ less when the left atrial catheter was removed at temperature of $36^{\circ} \mathrm{C}$.

T A B L E

\begin{tabular}{|c|c|c|c|}
\hline No. & Age & Sex & $\begin{array}{l}\text { Cardiac } \\
\text { Lesion }\end{array}$ \\
\hline 3 & 10 & $\mathbf{M}$ & V.S.D. \\
\hline 4 & 29 & $\mathrm{~F}$ & $\begin{array}{l}\text { A.S.D. } \\
\text { mitral defect }\end{array}$ \\
\hline 5 & 32 & $\mathbf{M}$ & V.S.D. \\
\hline 6 & 13 & $\mathbf{M}$ & A.S.D. \\
\hline 7 & 51 & $\mathbf{M}$ & Aortic \\
\hline 8 & 44 & $\mathrm{~F}$ & $\begin{array}{l}\text { Mitral } \\
\text { stenosis }\end{array}$ \\
\hline 9 & 14 & $\mathrm{~F}$ & V.S.D. \\
\hline 10 & 45 & $\mathrm{~F}$ & $\begin{array}{l}\text { Anomalous } \\
\text { pulmonary } \\
\text { veins }\end{array}$ \\
\hline 11 & 49 & M & $\begin{array}{l}\text { Aortic } \\
\text { stenosis + } \\
\text { incompetence }\end{array}$ \\
\hline
\end{tabular}

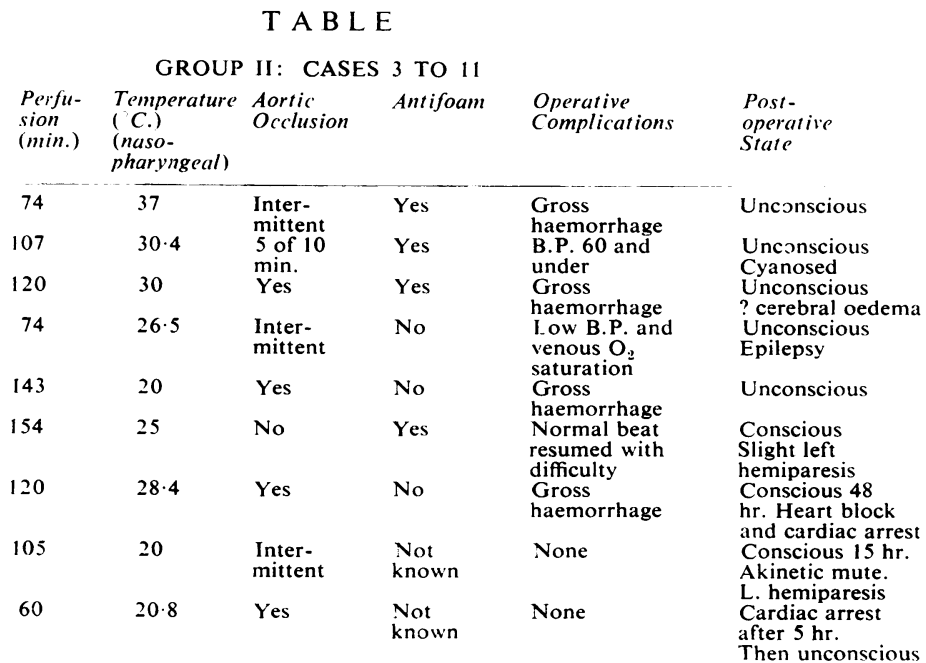


Post-operative course Consciousness was not regained and no pulses were felt. Spontaneous respiration returned after one and a half hours. There was no change until death after $10 \frac{1}{2}$ hours.

Necropsy Half a pint of fluid blood was found in each pleural cavity.

Macroscopic examination of the brain showed no internal herniae, and the basal vessels and ventricles were normal. Sharply defined areas of pallor involved from part to the whole of the cortical thickness and were most noticeable in the occipital lobes where petechiae were also present in the white matter.

Microscopic examination of the brain showed changes symmetrical in the hemispheres and maximal in the occipital lobes with involvement of the cortex and white matter. In the former, typical geographical lesions (Fig. 5) contained ischaemic neurones but no glial reaction. The blood vessels were normal apart from a single embolus of birefringent material, identified as Teflon and probably derived from the patch used to repair the septal defect (Fig. 6), and occasional fine globules of fat in cortical capillaries.

Geographical lesions were less marked in the temporal and minimal in the frontal lobes. In the Ammon's horns, only a few groups of cells showed ischaemic change. A few areas of pallor were seen in the caudate nucleus and putamen, and the thalami contained numerous small 'palings' particularly in the posterior half.

There was patchy pallor of myelin staining, greatest in the occipital lobe and involving the visual radiation (Fig. 7).

The brain stem was normal but the cerebellum showed localized lesions similar to those seen in cases 2 and 4.

CASE 5 (Maudsley No. 1688) A man aged 32 had a ventricular septal defect.

Operation Perfusion was carried out for $120 \mathrm{~min}$. at a minimal temperature of $30^{\circ} \mathrm{C}$. Ventricular fibrillation occurred when the aorta was occluded and 'haemorrhage was tremendous' when it was released. The perfusion rate was reduced to $50 \%$ and the mean blood pressure was $35-45 \mathrm{~mm}$. $\mathrm{Hg}$.

Post-operative course Consciousness was not regained, respiration was feeble, and the blood pressure was $110 / 70 \mathrm{~mm}$. $\mathrm{Hg}$. Blood transfusion and assisted respiration were commenced. The plantar responses were absent and, as cerebral oedema was suspected, intravenous dextrose was given. The patient was then regarded as decerebrate and death took place after $16 \frac{1}{2}$ hours.

Necropsy There was $25 \mathrm{oz}$. of unclotted blood in the right pleural cavity and $10 \mathrm{oz}$. in the left. Gastric perforations and erosions were noted.

Macroscopic examination of the brain showed some fullness of the gyri but no internal herniae. A thin film of subarachnoid blood covered the vertex of each hemisphere. The basal vessels and ventricles were normal. Numerous quadrilateral or irregular pale areas were seen in the lateral occipital cortex and in the parietal and temporal lobes (Fig. 8). A few petechiae in the occipital white matter were the only other abnormalities.

Microscopic examination of the brain showed focal and geographical lesions which were especially obvious in the occipital lobes and involved the calcarine regions. Ischaemic cell change was present in the lesions and in a small wedge of neurones in the Ammon's horns. Focal lesions were present in the caudate nucleus and putamen and were numerous in the posterior thalamus.

Localized lesions, as seen in cases 2 and 4, involved groups of folia on the inferior aspect of the cerebellum (Fig. 9).

The brain stem was normal. Large globules of fat were seen in a few cortical arterioles and some finer globules in occasional capillaries, but there was no associated tissue necrosis.

CASE 6 (Maudsley No. 1794) A boy aged 13 had an atrial septal defect (ostium primum) and cleft mitral valve.

Operation Perfusion was carried out for $74 \mathrm{~min}$. at a minimum temperature of $26.5^{\circ} \mathrm{C}$. The aorta was not clamped during the mitral valve repair and, as the atrium was partly empty, air embolism could have occurred. There was hypotension at the end of perfusion and the venous oxygen saturation was $6 \%$.

Post-operative course Consciousness was not regained and respiration was irregular. There was evidence of a mild right hemiparesis and there were periods of sudden and profound hypotension. Assisted respiration was necessary and surface cooling was begun. A right-sided Jacksonian fit was followed by several major seizures. Death occurred after 42 hours.

Necropsy There was patchy basal consolidation in both lungs. Bilateral subdural haematomas (up to 0.5 $\mathrm{cm}$. thick) were present over the occipital poles and in the floors of the middle and posterior fossae.

Macroscopic examination of the brain showed some congestion and flattening over the posterior half of the hemispheres. The basal vessels and ventricles were normal. Haemorrhagic and also well-defined pale areas were frequent in the right parieto-occipital region and sparse in the frontal lobe.

Microscopic examination of the brain showed focal and geographical lesions, obvious in the right parietooccipital region, which were also seen in the first temporal gyrus and in the ventro-lateral part of the frontal lobe. Petechial haemorrhages were numerous in these areas.

A single group of ischaemic neurones was seen in one Ammon's horn.

Patches of myelin pallor were sharply defined in the occipital and frontal lobes, and there was diffuse pallor in the corresponding digitate white matter.

In the basal ganglia there was a single focus of pallor in the putamen; the brain stem was normal while the cerebellum showed localized lesions similar to those seen in cases 2,4 , and 5 . There was no evidence of thrombosis in the cerebral vessels, but several cortical arterioles in a single frozen section contained a few globules of fat. 

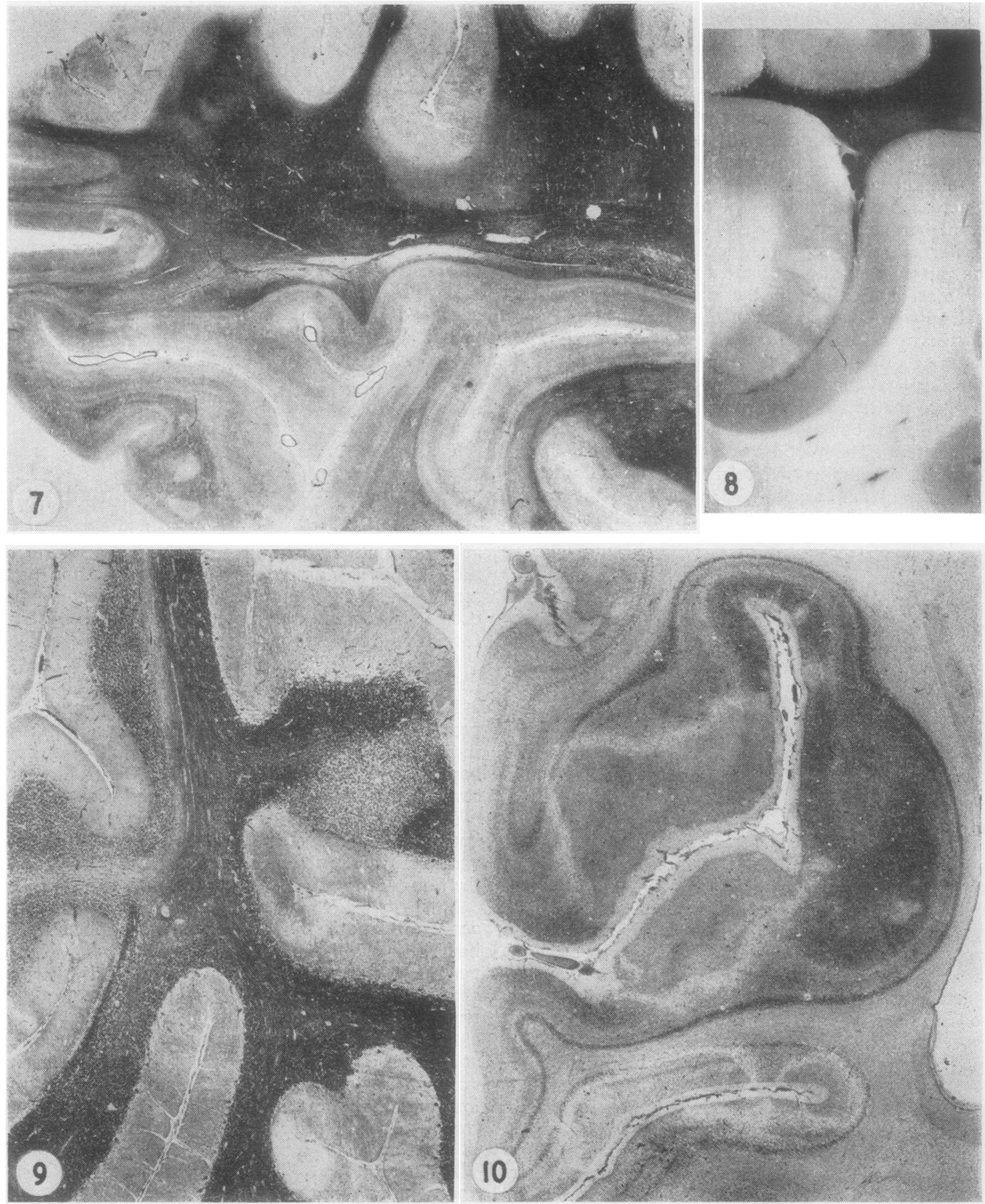

FIG. 7. Case 4. Occipital lobe. Horizontal section showing irregular areas of myelin pallor. (Heidenhain haematoxylin, $\times 2 \cdot 5)$

FIG. 8. Case 5. Brain slice. Occipital lobe shows sharply defined pale areas in cortex. $(\times 3)$

1TG. 9. Case 5. Cerebellum, showing irregular pallor of staining in the molecular and granular layers. (P.A.S. and haematoxylin, $\times 20$ )

FIG. 10. Case 9. Occipital cortex showing geographical areas of pallor surrounded by spongy borders. (Cresyl violet, $\times 5)$ 
CASE 7 (Maudsley No. 1806) A man aged 51 had calcific aortic stenosis.

Operation Perfusion was carried out for $143 \mathrm{~min}$. at a minimum temperature of $20^{\circ} \mathrm{C}$. Venous oxygen saturation was $23 \%$ to $25 \%$ before perfusion and the systolic blood pressure $50 \mathrm{~mm}$. Hg. Aortic valvotomy was performed and there was considerable blood loss throughout.

Post-operative course Consciousness was not regained. The blood pressure was $75 /$ ? $\mathrm{mm}$. Hg. Tracheostomy and assisted respiration were required. Nine pints of blood were transfused under pressure and concentrated platelets were given. There was little change until cardiac arrest occurred just before death after 48 hours.

Necropsy Twenty-four ounces of blood-stained fluid were found in the left pleural cavity and $16 \mathrm{oz}$. in the right. There was oedematous consolidation at the bases of both lungs.

Macroscopic examination of the brain showed no internal herniae and the basal vessels and ventricles were normal. Blotchy haemorrhagic areas were seen in the occipital cortex and the head of the left caudate nucleus.

Microscopic examination of the brain showed focal and haemorrhagic lesions, numerous on the lateral aspect of the occipital lobe and fewer in the temporal lobe. Petechial haemorrhages were abundant in all lesions.

The Ammon's horns showed a few ischaemic cells. Perivascular palings and petechiae were numerous in the caudate nuclei.

Myelin damage, largely in the occipital lobes, took the form of irregular areas of pallor. The brain stem was normal and the cerebellum showed lesions as seen in cases $2,4,5$, and 6 . The cerebral vessels showed no evidence of thrombosis and only an occasional fine fat droplet in a few cortical capillaries.

CASE 8 (Maudsley No. 1343) A woman aged 44 had mitral stenosis and regurgitation. She had had a closed mitral valvotomy five years previously.

Operation Perfusion was carried out for $154 \mathrm{~min}$. at a minimum temperature of $25^{\circ} \mathrm{C}$. The stenosis was relieved but not the incompetence. The valve was heavily calcified. Normal rhythm was established with difficulty.

Post-operative course The patient was conscious but there was 'poor circulation'. Tracheostomy and ass:sted respiration were required. Anuria occurred on the second post-operative day and spasticity was noted, more so on the left. There was deterioration to death on the third post-operative day.

Necropsy Some antemortem thrombus was found in the left auricular appendage and there was bilateral basal pulmonary consolidation.

Macroscopic examination of the brain showed no internal herniae, and the basal vessels and ventricles were normal. A few recent softenings up to $8.0 \mathrm{~mm}$. in diameter were seen in the centrum ovale and a few small palings in the lateral occipital cortex.
Microscopic examination of the brain showed geographical lesions only in the lateral occipital cortex. There were numerous microinfarcts (up to $3.0 \mathrm{~mm}$. in diameter) in the cortex, white matter, basal ganglia, and brain stem. These were probably caused by antifoam, as material stained by Oil Red $O$ but not by osmic acid was seen in the lumen of the vessels within some of them.

There were a few ischaemic neurones in the Ammon's horns, and in the ventral cerebellum were several lesions of the type seen in cases 2 and 4-7.

Myelin damage was present beneath the larger cortical lesions.

CASE 9 (Maudsley No. 1798) A girl aged 14 had a ventricular septal defect.

Operation Perfusion was carried out for $120 \mathrm{~min}$. at a minimum temperature of $28.4^{\circ} \mathrm{C}$. The septal defect was repaired by suture after considerable blood loss through the defect had been controlled by aortic and pulmonary clamps. The perfusion pressure fell to $50 \mathrm{~mm}$. $\mathrm{Hg}$ during the second hour.

Post-operative course Consciousness was regained fairly rapidly but the respirator was required for the first 24 hours. Later, when the respiration rate rose, nursing in an oxygen tent was begun. On the second post-operative day tracheostomy was carried out and the patient was placed on a respirator. On the third post-operative day $2: 1$ heart block occurred and the blood pressure fell to $85 / 40 \mathrm{~mm}$. $\mathrm{Hg}$. Cardiac arrest supervened, thoracotomy was carried out, and cardiac massage was begun within one and a half minutes. Pacemaker wires were inserted. The patient remained unconscious until death 23 hours after cardiac arrest and three and a half days from the end of perfusion.

Necropsy A persistent ductus arteriosus was found and there was purulent bronchitis and paralytic ileus.

Macroscopic examination of the brain showed no internal herniae, and the basal vessels and ventricles were normal. Sharply-defined areas of pallor were confined to the occipital cortex and involved the calcarine region. Similar areas in the head of the caudate nucleus were the only other abnormality.

Microscopic examination of the brain showed the major pathology, confined to the occipital and posterior temporal lobes and cerebellum, to consist of focal and geographical lesions (Fig. 10). Within these there were a few petechiae and no glial proliferation.

The Ammon's horns were noteworthy in that the greater part of each Sommer sector constituted an area of pallor limited by a narrow spongy border. Minor areas of pallor were seen in the caudate nucleus and globus pallidus of each side. The brain stem was normal while in the cerebellum there were several localized lesions, as in previous cases. There was no evidence of thrombosis or embolism in the cerebral vessels.

CASE 10 (Maudsley No. 1202) A woman aged 45 had a congenital anomaly of the pulmonary venous drain- 

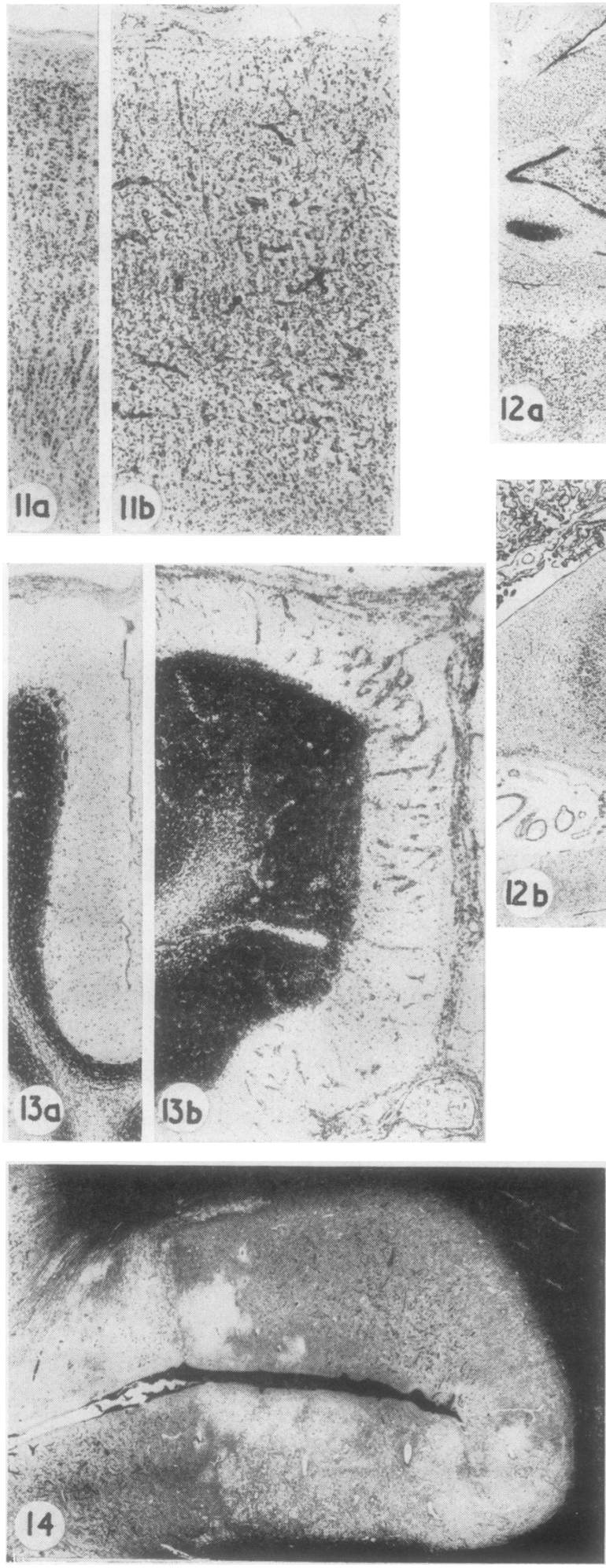
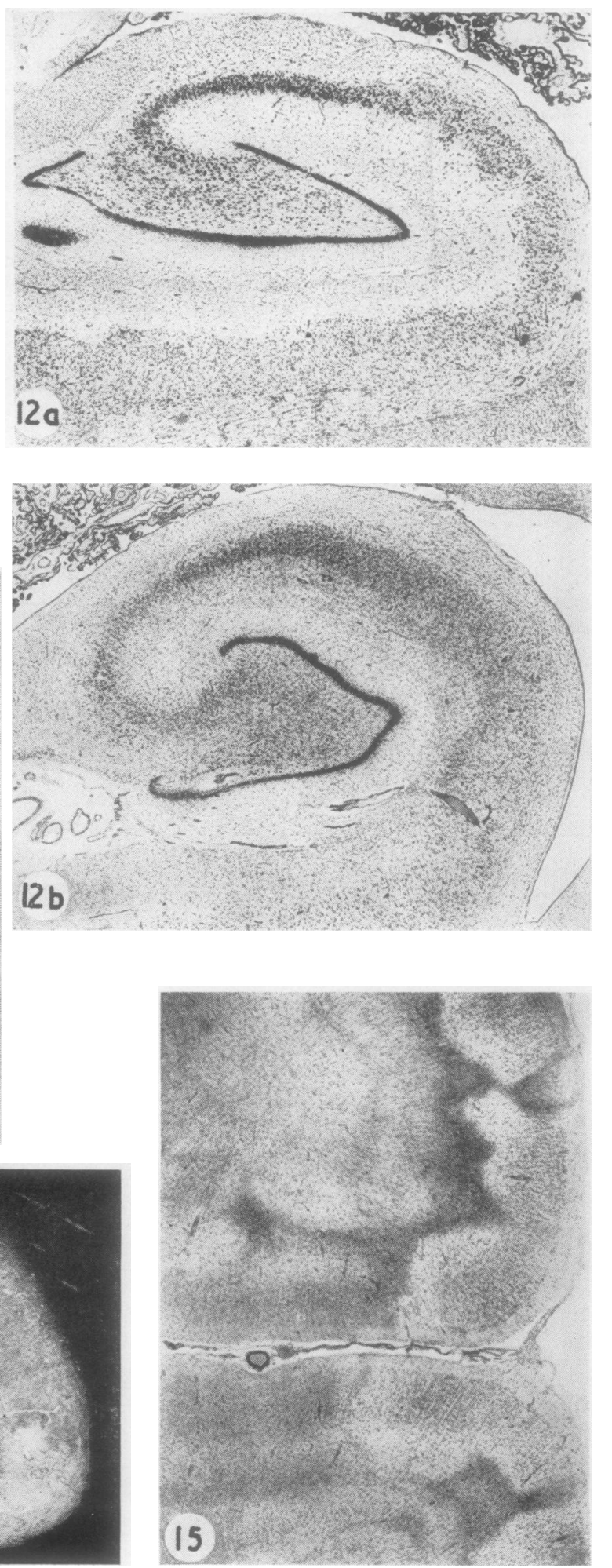


\section{LEGENDS TO PLATE OPPOSITE}

FIG. 11. (a) Normal occipital cortex. (Cresyl violet,$\times 35)$; (b) Case 11. Occipital cortex showing loss of neurones in all laminae and marked glial and capillary proliferation. (Cresyl violet,$\times 35)$

FIG. 12. (a) Normal Ammon's horn. (Cresyl violet, $\times 14)$; (b) Case 11. Ammon's horn. $(C r e s y l$ violet, $\times 14)$

FIG. 13. (a) Normal cerebellar cortex showing large Purkinje cells lying between the dark granular layer and the relatively cell-free molecular layer. (Cresyl violet, $\times 30)$; (b) Case 11. Cerebellar cortex showing loss of Purkinje cells with glial and capillary proliferation in molecular layer. (Cresyl violet, $\times 30)$

FIG. 14. Head injury, survival $10 \frac{1}{2}$ hours. Frontal cortex showing irregular areas of spongy tissue. (Heidenhain haematoxylin, $\times 9$ )

FIG. 15. Spontaneous subdural haematoma. Occipital lobe showing geographical areas of pallor in cortex. (Cresyl violet, $\times 13$ )

age (right and left pulmonary veins forming a common trunk entering the superior vena cava) and an atrial septal defect.

Operation Perfusion was carried out for $105 \mathrm{~min}$. at a minimum temperature of $20^{\circ} \mathrm{C}$. The anomalous channel was anastomosed to the left atrium and ligated in continuity; the atrial septal defect was closed. The heart restarted easily and the patient was conscious before leaving the theatre.

Post-operative course After 15 hours there were signs of pulmonary oedema. Tracheostomy and assisted respiration were required. The patient became akinetic and mute with a spastic left arm and leg and an extensor plantar response. There was slow deterioration to death on the fifth day.

Necropsy There was mild jaundice, a nutmeg liver, and haemorrhagic necrosis in both adrenals. An antemortem clot was seen in the stump of the ligated channel. A subdural haematoma (20 g.) lay over the left hemisphere.

Macroscopic examination of the brain showed a slight fullness of the left uncus, and the ventricles were rather small, particularly on the left. The basal vessels were normal.

Microscopic examination of the brain showed a few ill-defined lesions up to $1.0 \mathrm{~cm}$. in diameter in the occipital and temporal lobes. These showed capillary and glial proliferation with lipid phagocyte formation. A few small foci of cell loss were seen in the Ammon's horns, and there was a moderate diffuse cell loss and gliosis throughout diencephalon and cerebral cortex. Some focal lesions of the type seen in previous cases were noted in the ventral cerebellar cortex, and some of these were haemorrhagic. The brain stem was normal and no thrombi were found in the cerebral vessels. Small numbers of microinfarcts due to antifoam were seen in the cortex, basal ganglia, and white matter. The central white matter contained some softenings up to $2.5 \mathrm{~cm}$. in diameter which exhibited gliosis and lipid phagocyte formation.

CASE 11 (Maudsley No. 1338) A man aged 49 had aortic stenosis and incompetence.

Operation Perfusion was carried out for $60 \mathrm{~min}$. at a minimum temperature of $20.8^{\circ} \mathrm{C}$. The incompetence was corrected.

Post-operative course The patient was not fully conscious, was cyanosed and losing blood from the thoracic drainage tubes. A thoracotomy for removal of clot was carried out five hours after the end of perfusion. Cardiac arrest occurred as the chest was being closed (normal temperature). The chest was re-opened and cardiac massage begun after a delay of one and a half minutes. Normal beat was restored after seven minutes but the patient remained unconscious with extensor plantar responses until death on the eleventh post-operative day.

Necropsy Only histological data are available. There was some thrombosis and infarction in the spleen, glomerular hyalinization in the kidney, and a nutmeg liver.

Macroscopic examination of the brain showed no internal herniae, and the basal vessels and ventricles were normal. Poor anatomical definition in the thalami and Ammon's horns was the only other abnormality.

Microscopic examination of the brain showed changes which were diffuse in the hemispheres, cerebellum, and basal ganglia. In the cerebral cortex, damage was heaviest on the lateral aspect of the occipital lobes and least in the hippocampal and cingulate gyri. It was more obvious in the third layer and also in the walls and floors of the sulci (Fig. 11b). There was a brisk glio-mesodermal reaction with lipid phagocyte formation.

A typical anoxic necrosis of the Sommer sector together with cell loss in the end-folium were noted in the Ammon's horns (Fig. 12b). Cell loss and gliosis were heavy in the thalamus, caudate nucleus, and putamen. White matter damage appeared as scattered foci of demyelination in the centra semiovale, probably due to antifoam.

Cerebellar Purkinje cell loss was heavy and diffuse, more so on the dorsal aspect, and associated with proliferation of the Bergmann glia and of capillaries in the molecular layer (Fig. 13b). In the brain stem there was heavy gliosis around the aqueduct and in the floor of the fourth ventricle while cell loss was confined to the inferior colliculi and olives.

There was no evidence of antemortem thrombosis or of fat embolism in the cerebral vessels.

\section{SUMMARY OF PATHOLOGICAL FINDINGS}

Changes were localized rather than diffuse in the two cases of group I and in all but the last (case 11) of group II.

Localized lesions (except for case 10) consisted of focal (perivascular) and 'geographical' (irregu- 
lar) areas of neuronal pallor and/or loss outlined in most cases by a narrow band of spongy tissue.

Within these lesions, ischaemic cell change was recognizable after six and a half hours but glial reaction only from 48 hours onwards.

Cortical damage, heaviest in the posterior part of the brain in all cases, was greatest within the posterior part of the middle cerebral artery territory and in the cerebellum in four cases $(2,6,7$, and 8 ).

In three cases $(1,3$, and 9) the posterior as well as the middle cerebral artery territory was involved and there were localized cerebellar lesions in the last two.

The territories of all three cerebral arteries together with the cerebellum showed localized lesions in two cases (4 and 5). Localized lesions were seen in the basal ganglia of seven cases $(1,2$, $4,5,6,7$, and 9) being in the caudate nucleus in six, in the putamen in five, and in the thalamus in two.

Ischaemic lesions were seen in the Ammon's horn in cases 1 to 10 and were minor apart from cases 5 and 9 where they were large and of geographical type.

Focal and geographical lesions were not seen in the brain stem.

In case 10 , the cortical and white matter lesions were probably infarcts due to emboli from the clot in the ligated stump of the anomalous venous channel.

In case 11 , the changes were diffuse, involving cerebral and cerebellar cortex, Ammon's horn, diencephalon, and brain stem.

The white matter was normal only in case 9 and contained typical infarcts in case 10. Major damage was seen in cases $1,4,5,6$, and 7 as areas of blotchy myelin pallor, often related to cortical lesions and thus usually most severe in the occipital lobe. Damage was minor in cases 2, 3, 8 , and 11 , and appeared as a diffuse pallor or as irregular or perivascular foci of pallor.

Antifoam was responsible for microinfarcts in cases 8 and 10 and probably in case 11 .

Fat was seen in a few arterioles of cases 5 and 6 and in scattered groups of capillaries in cases 4,5 , 6 , and 7.

Subdural haemorrhage was seen in case 6 over both temporo-occipital regions (the cortical lesions were heamorrhagic), and in case 10 over the left hemisphere (ring haemorrhages were seen in the cerebellum and there was haemorrhagic necrosis in the adrenals).

Subarachnoid haemorrhage was seen in case 1 over the right occipital lobe (there was a small intracerebral haemorrhage and also altered blood in the intestines), and in case 5 over the vertex of both hemispheres (ring haemorrhages were seen in the cortex and there was fluid blood in the pleural cavities).

Thus subarachnoid and subdural haemorrhages were accompanied by evidence of haemorrhage in the brain itself and/or in the viscera.

\section{DISCUSSION}

In this series of 11 cases, cerebral damage was severe in cases 1-7 and 11, and only the latter regained consciousness after the operation. Damage was moderate in cases 8 and 9 and slight in case 10 , and all three were conscious for a variable period after the operation.

In case 10 the focal lesions were typical infarcts in the grey and white matter and almost certainly due to emboli from a clot in the ligated stump of an anomalous vessel. Case 11 was the only one to present the typical neuropathological picture of cerebral anoxia, due in this instance to postoperative cardiac arrest at normal body temperature.

The focal and geographical lesions were found in cases 1 to 9 ; case 1 survived 10 days and the remainder from six and a half hours to three and a half days.

Björk and Hultquist (1960), in their group of eight children dying after cardiac surgery, described diffuse cell loss in the cortex and globus pallidus of seven. Of these, six were submitted to deep hypothermia (range 5.8 to $11.0^{\circ} \mathrm{C}$. - oesophageal) which was obtained by the method of Drew and Anderson (1959) using the lungs as oxygenator. One patient perfused at normal temperature (but with coronary cooling) and surviving 21 days showed only minor changes in the globus pallidus and cortex. Only in their case 9 were lesions of clearly focal and geographical type described and illustrated. This was a child aged 4 years with a ventricular septal defect, who had a circulatory arrest of $37 \mathrm{~min}$. at a temperature of $16^{\circ} \mathrm{C}$. (oesophageal) during perfusion by a pump oxygenator. Survival was $\mathbf{3 8}$ hours.

If this case is added to the nine of the present series showing similar focal and geographical lesions, it may be concluded that these occur (1) in the age range $5-51$ years; (2) in the temperature range $37-16^{\circ} \mathrm{C}$. ; (3) with and without perfusion ; (4) with circulatory arrest ; (5) without circulatory arrest but in the presence of severely reduced cerebral blood flow during or just after perfusion ; (6) predominantly in the posterior half of the brain ; and (7) largely in grey but also in white matter. 
The focal character of the lesions in the white and grey matter and the presence of ischaemic cell change in the latter point unequivocally to ischaemia as their cause. The possible origins of such ischaemia can best be discussed in the context of the several factors that have been invoked to explain involvement of the central nervous system after open operations on the heart.

ANOXIA The focal and geographical lesions have neither the character nor the distribution of those due to such generalized anoxic episodes as circulatory arrest at normal temperature, hypoglycaemia or carbon monoxide poisoning. The pathology typical of anoxia is, in fact, seen only in case 11 (post-operative cardiac arrest at normal body temperature) and consists of diffuse neuronal loss in the cerebral cortex (Fig 11b), necrosis in the Sommer sector of the Ammon's horn (Fig. 12b), diffuse loss of cerebellar Purkinje cells (Fig. 13b), and widespread neuronal loss in the diencephalon.

This characteristic picture was not reported in the series of Björk and Hultquist or elsewhere in the available literature.

CEREBRAL OEDEMA This was regarded as a possible cause of neurological complications by Ehrenhaft and Claman (1961) and by Ehrenhaft, Claman, Layton, and Zimmerman (1961), who from a limited pathological examination of three brains interpreted cortical changes as evidence of oedema due to air embolism, inadequate perfusion or anoxia. It is known, however, that cerebral oedema is essentially an abnormality of the white matter (Greenfield, 1939) and is associated with cortical fullness and flattening, internal herniae, small lateral ventricles, and expansion of the white matter itself. These changes were not seen in the cases of Ehrenhaft et al. (1961) or in those of the present series (except one hemisphere of case 1 ; survival 10 days).

The oedema associated with cerebral tumour, abscess or trauma does not usually result in the localized lesions now under discussion and there is in fact no convincing evidence that oedema plays a part in their genesis.

EMBOLISM During the course of cardiac surgery emboli of several types may enter the blood stream.

Air Air may enter the circulation in at least four ways: by its inclusion as the heart is being closed; by the application of the defibrillator before the heart has been emptied of air ; by the production of bubbles (foaming) in the oxy- genator; and by the release of oxygen from cold blood while it is being rewarmed in the heat exchanger. Air embolism is a greater risk in operations on the left side of the heart and in the presence of septal defects. Cases 1 to 9, presenting localized cerebral pathology, are made up of three atrial septal defects, three ventricular septal defects, one aortic and one mitral stenosis, and one pulmonary stenosis (without septal defect). The latter is of particular interest in that it presented focal and geographical lesions to a severe degree.

Recently, Sloan, Morris, Mackenzie, and Stern (1962) have reported a series of 600 cases of cardiac surgery with 78 deaths. Air embolism was held responsible for mild neurological symptoms which cleared within a few days in most cases and within a week or two in a minority. In the absence of any account of the cerebral pathology in the fatal cases the true incidence and pathological significance of air embolism cannot be assessed.

The cerebral pathology resulting from intravascular air consists of perivascular areas of neuronal pallor and destruction which may be more or less confluent and may occur in cortex, basal ganglia, and cerebellum but are rare in the brain stem (Experimental: Spielmeyer, 1913; Bodechtel and Müller, 1930. Human : Neubürger, 1925 ; Lhermitte and Barrelet, 1934; Schulman, 1954).

Air might be expected to enter the uppermost vascular territory which, in the supine position, would be that of the anterior cerebral artery. In the present series, however, the localized lesions tend to lie in the posterior half of the brain.

It must be stressed that a categorical diagnosis of air embolism cannot be made from a morbid anatomical examination of the brain, and it can only be said that these localized lesions are compatible with an aetiology of air embolism. It is only in case 6 that this possibility was suspected during the operation.

Fat Fat embolism may result from the median or transverse sternotomy carried out to gain access to the heart and also from the mediastinal fat, globules of which may drain into the pericardial sac and be sucked into the perfusion system (Wright, 1963).

The cerebral pathology of fat embolism, as seen typically after fracture of the long bones, consists of perivascular haemorrhagic infarction of the white matter (often recognizable on naked-eye examination) and lesser foci of necrosis in the grey matter. This picture has not yet been described in patients dying after cardiac surgery, but intravascular sudanophilic material associated with 
small focal lesions was noted in four out of 14 cases by Bleifeld (1961).

Miller, Fonkalsrud, Latta, and Maloney (1962) claim that all patients who die within a week of cardiac surgery, in which extra-corporeal circulation was employed, exhibit fat embolism. Material stained by Oil Red $O$ was seen in capillaries and some arterioles. This finding was twice as obvious with median as with transverse sternotomy. Trauma to blood as it passed through the oxygenator was also suggested as a source of fat. No evidence was presented to show that these emboli resulted in cerebral damage and thus contributed to death. In the present series intravascular fat was only identified with certainty in an occasional arteriole of cases 5 and 6 and in scattered capillaries in cases 4 and 7 , and these findings were unrelated to tissue damage. Thus, fat embolism cannot be held responsible for the focal and geographical types of lesion.

Antifoam The cerebral lesions that are known to result from silicone antifoam emboli consist of microinfarcts up to $3 \mathrm{~mm}$. in diameter which are more numerous in the grey than in the white matter and are seen in the cortex, basal ganglia, brain stem, cerebellum, and white matter. In the present series, antifoam was employed in only three of the nine cases that showed localized lesions. The latter by virtue of their size, nature, and distribution are readily distinguished from those due to antifoam, and examples of the latter were present in two cases in the series (cases 8 and $10)$ and were doubtful in a third (case 11).

CIRCULATORY ARREST AND HYPOTENSION At an oesophageal temperature of $30^{\circ} \mathrm{C}$. the upper limit of circulatory arrest without cerebral damage is believed to be 8 to 10 minutes (Drew, 1961). The cerebral pathology resulting from a transgression of these limits does not appear to have been described in the human or experimental animal brain.

In view of the above, the clinico-pathological picture in case 1 is interesting, if inexplicable, in that a circulatory arrest of only $4 \mathrm{~min}$. $20 \mathrm{sec}$. at a temperature of $31.8^{\circ} \mathrm{C}$. was followed by clear evidence of brain damage as the patient left the theatre. In contrast to the effects of circulatory arrest at normal temperature (case 11) the pathology, although severe, was of localized type, and it is clear that only portions of the brain suffered severe ischaemia. It is quite possible, however, that such ischaemia may have occurred, not during the deliberate circulatory arrest but immediately thereafter, because some factor such as vasospasm or sludging of blood had prevented the re-establishment of normal flow within certain vessels.

In case 2, a circulatory arrest of $5 \mathrm{~min} .37 \mathrm{sec}$. at a temperature of $28.8^{\circ} \mathrm{C}$. was followed by ventricular fibrillation requiring cardiac massage for $60 \mathrm{~min}$. and thus adding a factor of hypotension of unknown degree. Once again the pathology was one of localized ischaemic rather than diffuse anoxic changes.

Severe arterial hypotension occurred in seven (cases 2, 3, 4, 5, 6, 7, and 9) of the nine cases showing localized lesions and was due to haemorrhage, low perfusion pressure or poor postoperative cardiac output (as indicated by a venous oxygen saturation of $6 \%$ in case 6 ).

Case 9 was conscious for the first two and a half days but hypotension due to heart block occurred on the third day, followed after a few hours by a cardiac arrest of one and a half minutes, and death took place 23 hours later. The absence of any glial or capillary proliferation in the geographical lesions suggests that they are unlikely to date from the time of the operation. As the patient was also conscious for the first two and a half days, the hypotensive episode seems the most probable cause of the cerebral pathology, and the cardiac arrest must have been of too short duration to have produced the diffuse anoxic pathology seen in case 11 .

Little is known of the neuropathological sequelae of hypotension at either reduced or normal temperature. In the human, Brierley and Cooper (1962) reported a single patient in whom drug-induced hypotension resulted in dementia and a cerebral pathology that was diffuse in both cerebrum and cerebellum. In a large series of Rhesus monkeys, Ingraham and Bering (1959) produced severe hypotension by a combination of Arfonad and blood loss. Cerebral pathology was seen in only one animal and consisted of sharply demarcated areas of cell loss together with considerable diffuse loss of cerebellar Purkinje cells while the Ammon's horns were normal.

Burton (1958) has shown that as blood pressure falls the flow within arterioles will cease at a particular pressure. At this 'critical closing pressure' the elastic tension in the vessel wall exceeds the hydrodynamic pressure within the lumen, and blood flow ceases. While there are now considerable data on this phenomenon in the somatic vessels, the 'critical closing pressure' within the cerebral vessels is not known and such closure has yet to be related to a definite cerebral pathology. 
Until further clinical, pathological, and experimental information is available it can only be reported that severe hypotension associated with a greatly reduced cerebral blood flow is a factor present in a majority of cases showing focal and geographical lesions. There is the possibility that this reduced blood flow could result from myocardial ischaemia due to the entry of air into the coronary circulation. In this way the pathological pictures of air embolism and of severe hypotension could be combined in some cases of this series.

POSTURE The concentration of lesions in the posterior portion of the brain, including the cerebellum, suggests that a gravitational pooling of blood may occur in these regions in a subject lying in the supine position. Such pooling might occur not only during circulatory arrest but also during a period of hypotension. The consequent congestion of the vascular bed might delay the resumption of normal blood flow when the heart restarts or when the blood pressure rises. The resulting ischaemia by damaging vessel walls as well as parenchyma may partly explain the frequency of petechial haemorrhages in the posterior part of the brain.

THERMAL INJURY Drew (1961) has suggested that cerebral injury may occur from the too rapid cooling or rewarming of blood passing to the brain. The mechanism is unlikely to be relevant to the localized lesions which occurred not only in the case of Björk and Hultquist cooled to $16^{\circ} \mathrm{C}$. but also in case 3 of the present series where hypothermia was not employed. It is to be noted that the cerebral pathology of thermal injury has not yet been described.

The above factors have been selected for discussion as they appear to be the more important among the very many that may contribute to the cerebral damage occurring in a proportion of the patients submitted to open cardiac surgery.

The changes taking place in the blood as a result of its passage through the pump-oxygenator or of hypothermia cannot be considered in full, but the thrombocytopenia reported by Sharp, Excell, Salzman, and Torup (1961) and by Bloom (1961) is important in relation to the subdural, subarachnoid, intracerebral, and perivascular haemorrhages noted in the present series and also to the platelet and leucocyte thrombi seen in the cerebral vessels of case 9 of Björk and Hultquist. It was this brain that presented lesions of geographical type.

In the present series, it was only in the cases surviving six hours (case 3 ) and $10 \frac{1}{2}$ hours (case 4) that aggregates of leucocytes and P.A.S.-positive granular material were seen in an occasional cortical arteriole. As similar appearances have been noted in vessels within recent cortical infarcts (Meyer, 1958; and personal observations), it is possible that such aggregates may be the result rather than the cause of parenchymal damage.

Lesions of focal and geographical type have been encountered in situations other than cardiac surgery, for example in a case of head injury with cortical contusions (Meyer, 1941) (Fig. 14), in instances of subarachnoid haemorrhage due to cerebral aneurysm (Logue and Smith, 1962), and in a case of spontaneous subdural and subarachnoid haemorrhage in a young adult (Strich, 1962) (Fig. 15).

Thus, apart from antifoam embolism, there is no reason to conclude that the accidents of open cardiac surgery with and without perfusion and with and without hypothermia result in a unique cerebral pathology.

The provisional conclusion is advanced that, in the absence of pathological evidence of anoxia and oedema, severely reduced cerebral blood flow and air embolism are the two most probable causes of the focal and geographical lesions described above. Many additional factors may play a supplementary role, and, of these, changes in the formed elements and in the chemistry of the blood appear to be the most important.

It is apparent, however, that in a situation as physiologically complex as that of cardiac surgery, the ability of reduced blood flow or any other factor to produce cerebral pathology can only be satisfactorily proven in the experimental animal where the maximum control of other variables is possible.

Finally, the question arises, can a cerebral pathology of a type similar to that described, but of lesser degree, exist in the brains of a proportion of the survivors of this type of surgery and be responsible for transient or permanent neuropsychiatric symptoms? Suggestive evidence that this may be so lies in the fact that in some of the present cases the cerebral lesions were relatively minor and the cause of death clearly cardiovascular. A satisfactory answer, however, could only be obtained from an extended follow-up study with ultimate pathological examination of the brain.

\section{SUMMARY}

The neuropathological findings in 11 patients dying from six and a half hours to 11 days after open cardiac surgery are reported. 
Perfusion by pump oxygenator was employed in nine, at normal body temperature in one and from 30 to $20^{\circ} \mathrm{C}$. in eight.

In nine cases a localized cerebral pathology was seen, consisting of focal (perivascular) and irregular (geographical) areas of cell loss or pallor of staining. Variable patchy pallor of myelin staining was also seen.

In one case the pathology was that of white matter infarcts probably due to clot emboli.

The diffuse changes typical of cerebral anoxia were seen in one case (post-operative cardiac arrest).

Air embolism and hypotension with severely reduced cerebral blood flow appear to be the principal factors in the pathogenesis of the focal and geographical lesions.

I am greatly indebted to Sir Russell Brock and to Mr. M. F. O'Brien of the Brompton Hospital and to the members of the Department of Neuropathology in the Institute of Psychiatry for considerable valuable advice.

This study has been made possible by the generous co-operation of the surgical teams in the six cardiac surgery units concerned.

It is also a pleasure to acknowledge the technical assistance of A. R. Salliss, F.I.M.L.T., and Mrs. N. Chimovits as well as the invaluable photographic skill of Miss Pamela Dix.

The present report forms part of a wider investigation of the cerebral complications of extracorporeal circulation and hypothermia in man and in experimental animals and is being supported by a grant from the Medical Research Council.

\section{REFERENCES}

Björk, V. O., and Hultquist, G. (1960). Brain damage in children after deep hypothermia for open-heart surgery. Thorax, 15, 284

Bleifeld, W. (1961). Cerebrale "Fett" embolie nach Operationen mit der Herz-Lungen-Maschine. Thoraxchirurgie, 9, 12.

Bloom, A. L. (1961). Changes in blood after using an extracorporeal circulation. Brit. med. J., $2,16$.

Bodechtel, G., and Müller, G. (1930). Die geweblichen Veränderungen bei der experimentellen Gehirnembolie. Z. ges Neurol. Psychiat. (Orig.), 124. 764.
Brierley, J. B., and Cooper, J. E. (1962). Cerebral complications of hypotensive anaesthesia in a healthy adult. J. Neurol. Neurosurg. Psychiat., 25, 24.

Burton, A. C. (1958). Interrelation of physical and physiological factors. In Factors Regulating Blood Flow. Proc. 3rd Cont. (D) Microcirculatory Physiology and Pathology, Milwaukee, 1956. p. 3! . (1961). Profound hypothermia in cardiac surgery, Brit.

Drew, C. E. (1961). Profound hypothermia in cardiac surgery. Brit. med. Bull., 17, 37.

- and Anderson, I. M. (1959). Profound hypothermia in cardiac surgery. Report of three cases. Lancet, $i, 748$.

Ehrenhaft, J. L., and Claman, M. A. (1961). Cerebral complications of open-heart surgery. J. thorac. cardiovasc. Surg., 41, 503.

- Layton, J. M., and Zimmerman, G. R. (1961). Cerebral complications of open-heart surgery. Further observations. Ibid., 42, 514 .

Greenfield, J. G. (1939). The histology of cerebral oedema associated with intracranial tumours (with special reference to changes in the nerve fibres of the centrum ovale). Brain, 62, 129.

Ingraham, F. D., and Bering, E. A. (1959). Safe limits of hypothermia and hypotension. Progress report. United States Public Health Services Grant B-1175.

Lhermitte, J., and Barrelet (1934). Embolie gazeuse cśrébrale d'origine périphérique. Étude anatomique. Rev. neurol., 41 (2), 851.

Lindberg, D. A. B., Lucas, F. V., Sheagren, J., and Malm, J. R. (1961). Silicone embolization during clinical and experimental heart surgery employing a bubble oxygenator. Amer. J. Path., $39,129$.

Logue, V., and Smith, B. (1962). Cerebral pathology in ruptured intracranial aneurysms. Proc. Soc. Brit. Neurol. Surg. 65th meeting. In J. Neurol. Neurosurg. Psychiat., 25, 393.

Meyer, A. (1941). Unpublished case.

Meyer, J. S. (1958). Localized changes in properties of the blood and effects of anticoagulant drugs in experimental cerebral infarction. New Engl. J. Med., 258, 151 .

Miller, J. A., Fonkalsrud, E. W., Latta, H. L., and Maloney, J. V. (1962). Fat embolism associated with extracorporeal circulation and blood transfusion. Surgery, 51, 448 .

Neubürger, K. (1925). Über cerebrale Fett und Luftembolie (nebs I Bemerkungen zur Frage der Schichterkrankungen der Grosshirnrinde und der Pathogenese der Keuchhusteneklampsie der Kinder). Z. ges. Neurol. Psychiat. (Orig.), 95, 278.

Ross, D. N. (1954). Venous cooling. A new method of cooling the bloodstream. Lancet, $i, 1108$.

Schulman, S. (1954). Some uncommon acute neurological disorders Med. Clin. N. Amer., 38, 167.

Sharp, A A Excell, B., Salzman, E and Torup, O (1961). The haematology of extra-corporeal circulation with and without hypothermia. In Thrombosis and Anticoagulent Therapy, ed. W. Walker, pp. 88-93. Livingstone, Edinburgh.

Sloan, H., Morris, J. D., Mackenzie, J., and Stern, A. (1962). Open heart surgery; results in 600 cases. Thorax, 17, 128.

Smith, W. T. (1960). Cerebral lesions due to emboli of silicon anti-foam in dogs subjected to cardiopulmonary bypass. $J$. Path. Bact., 80, 9.

Spielmeyer, W. (1913). Üeber die anatomischen Folgen der Luftembolie ins Gehirn. Verh. dtsch. Kongr. inn. Med., 30, 359.

Strich, S. (1962). Unpublished case.

Thomassen, R. W., Howbert, J. P., Winn, D. F., and Thompson. S. W. (1961). The occurrence and characterization of emboli associated with the use of a silicone antifoaming agent. $J$. thorac. cardiovasc. Surg., 41, 611.

Wright, E. S. (1963). Blood damage in prolonged extracorporea circulation. Surgery (in the press)

Yates, P. O., Cassie, A. B., Dark, J. F., Jack, G. D., and Riddell, A. G (1959). The detection of antifoam emboli following perfusion with a heart-lung machine. Lancet, 1, 130. 